\title{
Choques na Taxa de Câmbio Real e o Saldo da Balança Comercial Agropecuária Brasileira: evidências da Curva J entre 1994 e 2007
}

\author{
Paulo Roberto Scalco ${ }^{1}$, Henrique Duarte Carvalho² e \\ Antonio Carvalho Campos ${ }^{3}$
}

Resumo: O objetivo deste trabalho é analisar os efeitos de curto e longo prazo de choques na taxa de câmbio real sobre o saldo da balança comercial agropecuária brasileira, após a implantação do Plano Real. O procedimento metodológico empregado foi a análise multivariada descrita em Johansen (1991). Os resultados indicam que existem duas relações de longo prazo entre o saldo da balança comercial agrícola e renda doméstica com relação à renda do estrangeiro e à taxa de câmbio real. Verificou-se que, no longo prazo, um aumento de $1 \%$ da taxa de câmbio e na renda externa leva à expansão de 2,04\% e 1,95\%, respectivamente, no saldo da balança comercial. Esse resultado é consistente com a condição de Marshall-Lerner, que afirma: no longo prazo, o efeito volume deve superar o efeito preço, aumentando, assim, o saldo da balança comercial. Entretanto, no curto prazo, rejeita-se a hipótese de existência da Curva J.

Palavras-chaves: Condição de Marshall-Lerner, Curva J, taxa de câmbio, balança comercial, setor agropecuário.

Abstract: The aim of this work is to analyze the effect of shocks in the real exchange rate in long and short-run on the Brazilian agricultural trade balance after the deployment of Plano Real. The used methodological framework was an application of Johansen's (1991) multivariate analysis. Results indicate that two relations of long-run exist between the agricultural trade balance and the domestic income regarding the foreign income and variations in the real exchange rate. It was verified that, in the long-run, an increase of $1 \%$ in the exchange rate and in the external income leads respectively to an expansion of $2.04 \%$ and $1.95 \%$ in the trade balance. Our empirical findings reveal that the Marshall-Lerner

\footnotetext{
1 Professor da Faculdade de Administração, Ciências Contábeis e Ciências Econômicas da Universidade Federal de Goiás (Face-UFG). E-mail: pauloscalco@yahoo.com.br

2 Professor de economia da Fundação Comunitária de Ensino Superior de Itabira (Funcesi). E-mail: henrique.carvalho@funcesi.br

3 Professor do Departamento de Economia Rural da Universidade Federal de Viçosa (DER-UFV). E-mail: accampos@ufv.br
} 
condition holds that, in the long-run, the quantum effects must surpass price effects, increasing the trade balance. However, in the short-run, the hypothesis of an existence of the J Curve was rejected.

Key-words: Marshall-Lerner condition, J Curve, exchange rate, trade balance, agricultural sector.

Classificação JEL: F13, Q17.

\section{Introdução}

Em face da instabilidade macroeconômica vivida nos anos 1980 e início dos anos 1990, o Brasil passou por períodos distintos e alterações significativas em suas políticas monetária, fiscal e cambial. Segundo Kannebley (2002), essas alterações estavam associadas, de modo mais ou menos direto, ao objetivo maior de estabilização da inflação e manutenção do equilíbrio externo da economia, compreendendo mudanças de regimes cambiais e regras de condução e administração tanto das taxas de câmbio quanto dos mercados cambiais.

Em consonância com o movimento mundial de liberalização, abertura de mercados e redução do papel do Estado na economia, diversas medidas foram adotadas no País a partir de 1990. A abertura do mercado brasileiro à concorrência dos importados refletia a intenção de estimular o aumento da eficiência produtiva das firmas nacionais, não por meio de incentivos ou subsídios oficiais, mas por meio da necessidade de sobrevivência no mercado. Sinalizava-se, assim, uma nova etapa na qual o País teria uma relação fundamentalmente diferente com o mercado internacional, por meio da maior integração tanto no comércio de bens e serviços quanto nos movimentos de capitais. Esta mudança no perfil das transações externas e a apreciação da nova moeda do País refletiram a mudança da relação da economia brasileira com o setor externo, com elevação rápida das importações e do déficit da balança comercial a partir de 1994.

A partir de 1995, atingida a estabilização dos preços com a implementação do Plano Real, foi introduzido o regime de bandas cambiais com o objetivo de incrementar a credibilidade da política cambial, por meio da adoção de medidas que implicavam maior estabilidade da taxa de câmbio real e do padrão de variação da taxa de câmbio nominal. Em janeiro de 1999, a manutenção deste regime tornou-se insustentável, levando o governo a adotar um regime de taxas flexíveis, o que produziu uma sensível depreciação nas taxas de câmbio nominal e real.

Observa-se, a partir de então, melhora significativa na balança comercial brasileira, creditada à desvalorização cambial; porém, grande parte desta melhora também pode ser atribuída à intensidade do comércio mundial, que demandou maiores quantidades de produtos a preços crescentes, sobretudo a partir de 2002, em função do acelerado crescimento da economia mundial.

Especificamente quanto ao setor agropecuário, verifica-se que foi penalizado pela predominância de uma taxa de câmbio valorizada na maior parte do período de adoção das bandas. Uma vez que o Brasil se caracteriza por ser um grande exportador de commodities, e não um importador, o saldo observado na balança comercial agropecuária, embora positivo na média, mostra-se irrisório com relação ao que se tornou logo após a desvalorização cambial (Figura 1). A manutenção da valorização pré-determinada reduziu a competitividade do setor frente ao mercado mundial e comprometeu significativamente a capacidade exportadora. A partir de 1999, entretanto, percebe-se que, com a flexibilização do mercado cambial, ocorre uma abrupta depreciação das taxas de câmbio e, consequentemente, o saldo comercial do setor agropecuário melhora. 
Figura 1. Saldo da balança comercial agropecuária (milhões de U\$\$) e taxa de câmbio comercial (R\$/U\$) - jan/90 a dez/07.

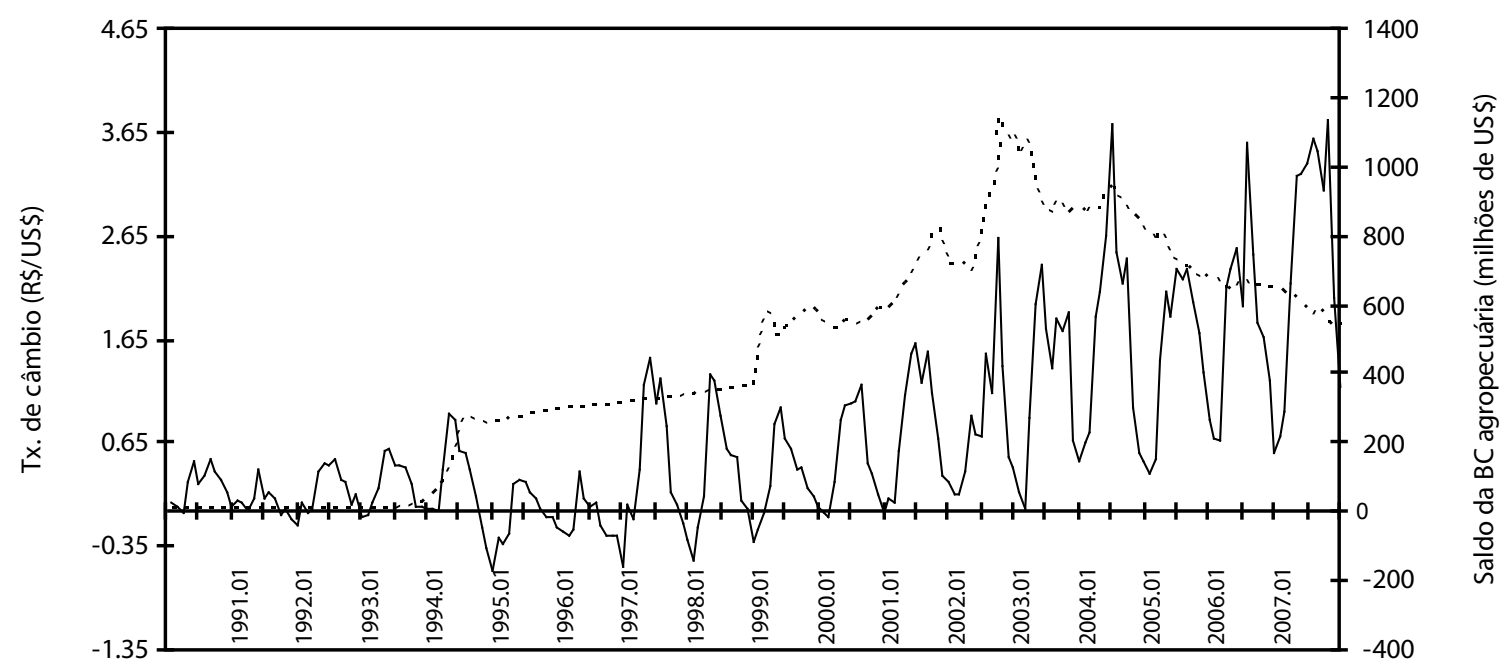

Fonte: Ipeadata.

Uma análise simplista dos efeitos da desvalorização cambial sobre a balança comercial, em um modelo de dois países, considerando-se o país estrangeiro como o resto do mundo, pode ser feita por

$$
B C=P X\left(Y^{*}, R\right)-E P^{*} Q(Y, R),
$$

em que $B C$ é o saldo da balança comercial, $P$ é o nível de preço do país local, $X(\bullet)$ é a demanda por exportações, $Q(\bullet)$ é a demanda por importações, $Y^{*}$ é a renda do resto do mundo, $R$ é a taxa de câmbio real $R=E P^{*} / P, E$ é a taxa de câmbio nominal, $P^{*}$ é o nível de preço do resto do mundo e $Y$ é a renda local.

$O$ efeito final da desvalorização sobre $B C$ desencadeia três processos: (i) aumento das exportações dado que a desvalorização cambial torna os bens domésticos mais baratos para o estrangeiro; (ii) aumento do preço doméstico das importações reduzindo o volume importado e (iii) a depreciação faz com que o país local tenha que pagar mais caro pelas importações remanescentes, o que deterioraria $B C$. Os efeitos (i) e (ii) são chamados de efeito volume e (iii) é o efeito preço. A implicação final da desvalorização sobre $B C$ depende de qual efeito predominará, sendo que ocorrerá melhora quando (i) e (ii) prevalecerem a (iii). Para que haja melhora de $B C$ é necessário que a derivada de (1) em relação a E (taxa de câmbio) seja positiva. Algebricamente tem-se:

$$
P \frac{\partial X}{\partial E}-E P * \frac{\partial Q}{\partial E}-p * Q>0
$$

Esta é a condição de Marshall-Lerner, segundo a qual somente haverá melhora na balança comercial decorrente de uma desvalorização cambial se, e somente se, o volume das exportações e das importações for elástico em relação à taxa de câmbio real. É esperado, deste modo, que desvalorizações nominais no câmbio provoquem uma melhora no saldo da balança comercial com o aumento das exportações e, destarte, a competitividade dos países no mercado mundial seria ampliada.

Contudo, o ajuste dos fluxos de comércio pode conter elementos dinâmicos que não conduzem ao ajustamento imediato do saldo da conta corrente. Este efeito de piora da conta corrente dos países logo após uma desvalorização nominal do câmbio é conhecido como "Curva J", em virtude dos efeitos perversos sobre o saldo da conta corrente no curto prazo e da melhora da posição ao longo do tempo, ou seja, os resultados, 
quando representados graficamente mostram um comportamento semelhante à letra J.

Teoricamente, este fenômeno ocorre devido ao lento ajuste nas quantidades demandadas e ofertadas às mudanças nos preços relativos. Assim, uma depreciação cambial, inicialmente, significaria exportações mais baratas e importações mais caras, fazendo com que o saldo da balança comercial piorasse. Depois de um tempo, o volume das exportações aumentaria por causa dos preços mais baixos para compradores estrangeiros e consumidores domésticos comprariam menos produtos importados, agora mais caros, melhorando a balança comercial (MOURA et al. 2005).

Segundo Yazici (2006), este comportamento é principalmente causado por respostas defasadas às mudanças na taxa de câmbio dos fluxos reais. No período da desvalorização/depreciação, as quantidades de exportações e importações são usualmente pré-determinadas por contratos realizados em períodos anteriores. Os volumes, portanto, responderão às mudanças na taxa de câmbio somente quando novos contratos forem assinados, e cujos efeitos são observados algum tempo depois da desvalorização/depreciação.

Muitos estudos têm estimado os efeitos de uma mudança na taxa de câmbio real sobre a balança comercial e confirmado a existência da Curva J. Carter et al. (1989) encontraram indícios empíricos da existência do primeiro segmento da Curva J para a balança comercial do setor agrícola americano. Doroodian et al. (1999) estimaram a Curva J para comércio agrícola e industrial dos EUA, e seus resultados confirmam a existência do efeito para o setor agrícola, porém, não para bens manufaturados. Kamoto (2006) estudou este fenômeno para Malawi e África do Sul, e seus resultados indicaram a existência da Curva J apenas para África do Sul. Ardalani et al. (2007) investigaram a existência da Curva J em nível industrial nos EUA desagregando os dados a um conjunto de 66 indústrias, das quais apenas seis apresentaram indícios empíricos confirmando a existência do efeito. Entretanto, Ahmad et al. (2004), ao estudar o comércio bilateral da China com países do G-7, não encontrou indícios de uma resposta negativa de curto prazo que caracterizasse a Curva J. Yazici (2006) chegou à mesma conclusão ao estudar o setor agrícola da Turquia. No Brasil, Moura et al. (2005) estimaram os efeitos da Curva J para dados agregados da balança comercial, como nos estudos anteriores, e os autores descartaram a existência do efeito para a balança comercial brasileira.

Nesse contexto, o propósito deste trabalho é estimar, através de uma abordagem de cointegração, os efeitos de curto e longo prazo de mudanças cambiais sobre a balança comercial do setor agropecuário brasileiro, após a implantação do Plano Real e, em específico, testar a existência do efeito da Curva J.

O restante do estudo está divido em quatro seções. A seção dois apresenta o modelo teórico, enquanto que na terceira seção são realizadas as análises das séries utilizadas, a estimação do modelo empírico e a apresentação dos resultados obtidos. Finalmente, na quarta e última seção são feitas as considerações finais.

\section{Modelo teórico}

Para o presente estudo, utilizou-se um modelo de substitutos imperfeitos empregado por Kamoto (2006) e desenvolvido originalmente por Goldestein et al. (1985), em que a balança comercial abrange somente as mercadorias exportadas e importadas. Renda doméstica e preços das importações são os principais determinantes da demanda de bens importados. Assim, essa relação pode ser expressa como:

$$
M_{d}=M_{d}\left(Y, P_{m}, P_{d}\right),
$$

em que: $M_{d}$ é a demanda de importações domésticas, $Y$ é a renda doméstica, $P_{m}$ é o preço pago pelas importações em moeda doméstica e $P_{d}$ é o nível de preço geral no país doméstico. Similarmente, a oferta de bens produzidos domesticamente (que equivale à demanda de exportações do estrangeiro) ao resto do mundo é expressa como: 


$$
X_{d}=X_{d}\left(Y^{*}, P_{x}, E, P_{f}\right)
$$

em que: $X_{d}$ é a quantidade de bens exportados ao resto do mundo, $Y^{*}$ é a renda externa, $P_{x}$ é o preço pago em moeda estrangeira por importadores domésticos, $P_{f}$ é o nível de preço geral no país estrangeiro e E é a taxa de câmbio nominal, definida como o número de unidades de moeda doméstica por unidade de moeda estrangeira. A suposição-chave nas equações (3) e (4) é a de que as elasticidades-renda tanto doméstica quanto do estrangeiro são positivas, assim como as elasticidades-preço cruzadas, enquanto a própria elasticidade-preço é negativa. Neste modelo, a variável demanda é representada pela renda corrente e não pela renda transitória ou permanente. Esta condição adotada por economistas assume homogeneidade da função de demanda. Como resultado, consumidores fazem suas decisões baseados em valores reais ao contrário de valores nominais (ilusão monetária). Em decorrência da suposição de homogeneidade, o lado direito das equações (3) e (4) é dividido por seus respectivos preços domésticos e as seguintes equações são derivadas:

$$
M_{d}=M_{d}\left(Y_{r}, R P_{m}\right)
$$

em que: $Y_{r}$ é a renda real doméstica e $R P_{m}$ é o preço relativo das importações; e

$$
X_{d}=X_{d}\left(Y_{r}^{*}, R P_{x}\right)
$$

em que: $Y_{r}^{*}$ é a renda real do estrangeiro e $R P_{x}$ é o preço relativo das exportações. Quando o preço em moeda estrangeira, das exportações do estrangeiro $P_{x}$, é ajustado pela taxa de câmbio, ele equivale ao preço relativo das importações domésticas, assim, pode-se derivar a seguinte equação:

$$
R P_{m}=\frac{P_{m}}{P_{d}}=\frac{E P_{x}}{P_{d}}=\frac{E P_{f}}{P_{d}} \frac{P_{x}}{P_{f}}=Q P_{x}^{*}
$$

em que: $P_{x}^{*}$ é o preço real, em moeda estrangeira, das exportações do estrangeiro e $Q$ é a taxa real de câmbio. Nessa formulação, um aumento em $Q$ está associado à depreciação da moeda doméstica. Uma vez que exportações domésticas são importações do estrangeiro e o contrário é verdadeiro, a demanda por importações domésticas é equivalente à oferta de exportações do estrangeiro e a oferta de exportações domésticas é equivalente à demanda de importações do estrangeiro. Assim,

$$
M_{d}=X_{s}^{*}, X_{d}=M_{s}^{*}
$$

em que: $X_{s}^{*}$ e $M_{s}^{*}$ são oferta de exportações do estrangeiro e oferta de importações do estrangeiro, respectivamente. Dessa forma, deriva-se a equação de longo prazo para a balança comercial, como

$$
T B=P_{x}^{*} X_{d}-Q M_{d}
$$

A balança comercial (TB) é a diferença entre o valor das exportações e importações. Um valor negativo na balança comercial implica déficit comercial e está associado ao aumento no valor das importações relativo às exportações. A interação das variáveis na equação (9) resulta na seguinte forma reduzida da equação em valores reais:

$$
\begin{aligned}
& T B=T B\left(Y, Y^{*}, Q\right), \\
& \frac{\partial T B}{\partial Y}<0, \frac{\partial T B}{\partial Y^{*}}>0, \frac{\partial T B}{\partial Q}>0
\end{aligned}
$$

A equação acima é a função keynesiana tradicional para a balança comercial, em que renda doméstica real, renda real do estrangeiro e a taxa de câmbio real são os principais determinantes das exportações líquidas.

\section{Modelo econométrico e resultados empíricos}

O modelo teórico serve para guiar a especificação de um modelo empírico. Na equação (9) definiu-se a balança comercial como a diferença entre o valor das exportações e importações. Neste estudo, a balança comercial do setor agropecuário é definida como a razão das exportações $(X)$ e importações $(M)$ do setor. Este estudo utiliza a forma reduzida da equação de Gupta-Kapoor et al. (1999) para investigar a existência da Curva J utilizando variáveis reais. Dessa forma, a balança comercial é uma função da renda doméstica, renda do estrangeiro e taxa de câmbio, de acordo com a especificação a seguir: 


$$
\begin{aligned}
& \ln (X / M)=\beta_{0}+\beta_{1} \ln Y+ \\
& +\beta_{2} \ln Y^{*+} \beta_{3} \ln R E R+\varepsilon
\end{aligned}
$$

em que: $\ln (X / M)$ é o logaritmo natural da razão $X / M, \ln Y$ é o logaritmo natural da renda doméstica real, $\ln Y^{*}$ é o logaritmo natural da renda do estrangeiro, $\ln R E R$ é o logaritmo natural da taxa de câmbio real efetiva, $\beta_{0,} \beta_{1}, \beta_{2}$ e $\beta_{3}$ são os parâmetro a serem estimados e $\varepsilon$ é o termo de erro (i.i.d.).

Dada a natureza das séries utilizadas, segue-se a abordagem do paradigma de modelagem de séries temporais não estacionárias. Primeiro são analisadas as propriedades temporais de cada série utilizada por meio do tradicional teste de Dickey-Fuller aumentado (ADF), dos testes de HEGY para presença de componentes sazonais nas séries e o teste sugerido em Lütkepohl et al. (2004) para presença de quebra estrutural nas séries. Logo após são determinadas as relações de longo prazo existentes entre as quatro variáveis no sistema. Finalmente é estimado o modelo de Vetor de Correção de Erros (VEC), que permitirá realizar as inferências de curto e longo prazo do sistema analisado.

O modelo VEC é construído utilizando-se a abordagem de estimação de Johansen (1991), consistente com estudos recentes (KAMOTO, 2006; YAZICI, 2006; MOURA et al. 2005; ARORA et al. 2003).

\subsection{Testes de Raiz Unitária}

As séries de dados mensais de exportações $(X)$ e importações $(M)$ do setor agropecuário, total das importações mundiais e taxa de câmbio real do setor agropecuário $(R E R)^{4}$ foram obtidas

4 A taxa de câmbio real do setor agropecuário é calculada pelo Ipea por meio de uma média ponderada do índice de paridade do poder de compra dos 15 maiores parceiros comerciais do setor agropecuário. A paridade do poder de compra é definida, entretanto, pelo quociente entre a taxa de câmbio nominal (em R\$/unidade de moeda estrangeira) e a relação entre o Índice de Preço ao Consumidor (INPC) do país em caso e o Índice Nacional de Preços ao Consumidor (INPC/IBGE) do Brasil. As ponderações utilizadas são as participações médias de cada parceiro no total do comércio bilateral (exportações mais importações) brasileiras do setor de 2000 a 2004. no site do Ipea (Instituto de Pesquisa Econômica Aplicada), sendo que o Produto Interno Bruto (PIB) brasileiro foi obtido no site do Banco Central do Brasil (Bacen). Todas as séries correspondem ao período entre julho de 1994 a dezembro de 2007, totalizando 162 observações.

O saldo da balança comercial da agropecuária é definido como a razão entre exportações e importações, $\mathrm{BP}=(\mathrm{X} / \mathrm{M})$. A vantagem desta abordagem é que, utilizando-se a razão entre as duas variáveis, não é necessário deflacionar as séries para torná-las em termos reais; além disso, em casos nos quais a balança for deficitária (importações maiores que exportações), por exemplo, o coeficiente da razão é menor que um, porém ainda maior que zero, o que possibilita o uso da variável em forma logaritma, permitindo a estimação de suas elasticidades de forma direta.

O PIB brasileiro é utilizado como proxy para renda nacional $(Y)$ e as importações mundiais, como proxy da renda do estrangeiro $\left(Y^{*}\right)$. A representação gráfica das séries utilizadas está na Figura 2, e todas as variáveis estão na forma de logaritmo.

O procedimento adotado para o teste de raiz unitária é sugerido por Doldado et al. (1990), que utiliza o teste de Dickey-Fuller Aumentado (ADF) para verificar se as séries possuem raiz unitária ou não (ENDERS, 1995). Contudo, como observado na Figura 2, as séries do logaritmo da balança comercial $(B P)$ e renda do estrangeiro $\left(Y^{*}\right)$ podem conter componentes sazonais, e as séries do logaritmo do PIB brasileiro $(Y)$ e da taxa de câmbio real (RER) podem conter quebras estruturais no período analisado. Essas características tornam-se importantes, pois sua presença interfere negativamente no poder do teste ao invalidar as pressuposições assumidas sobre o termo de erro (MADDALA et al., 1998). Desta forma, utilizou-se também os testes de HEGY para verificar a presença de raízes unitárias sazonais, e o procedimento sugerido em Lütkepohl et al. (2004) para o teste de raiz unitária na presença de quebra estrutural.

A Tabela 1, reporta os resultados dos testes ADF, HEGY e de quebra estrutural. Verifica-se, 
Figura 2. Séries balança comercial do setor agropecuário (BP), PIB brasileiro $(\mathrm{Y})$, importações mundiais $\left(\mathrm{Y}^{*}\right)$ e taxa de câmbio real (RER) - jul/1994 a dez/2007.

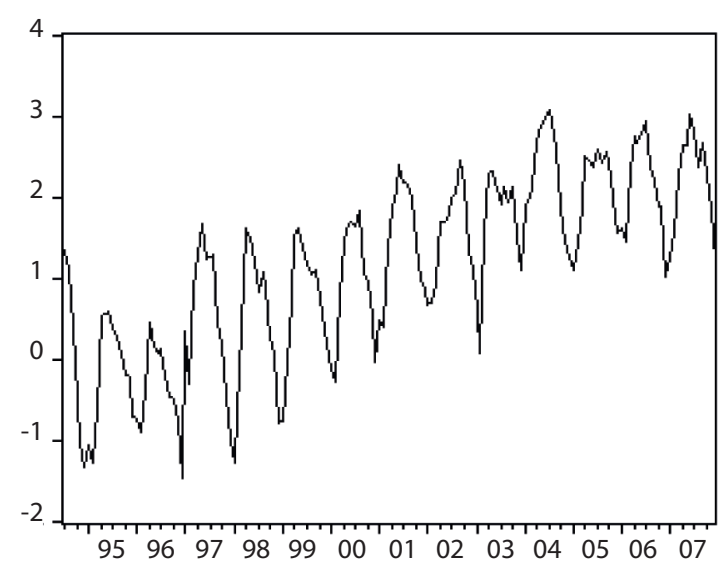

(a) BP

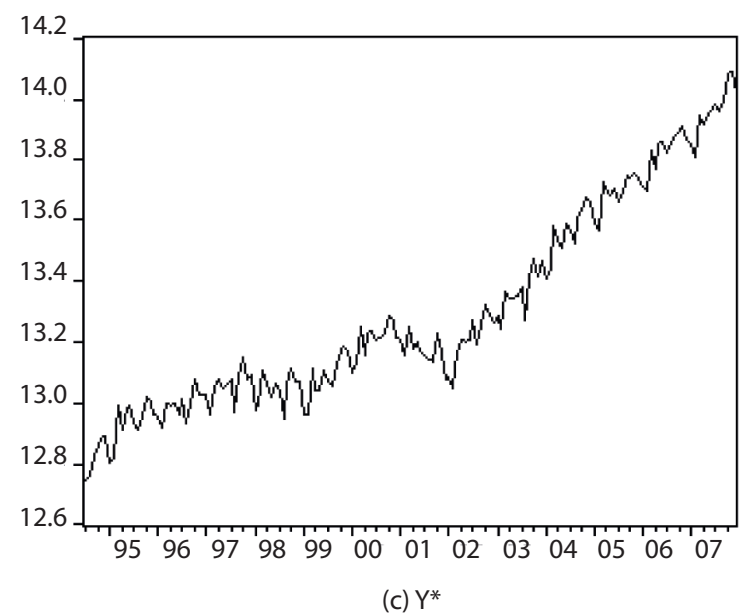

Fonte: Ipea e Bacen.

por meio do teste $\mathrm{ADF}$, que a hipótese nula de presença de raiz unitária não é rejeitada ao nível de significância de 1\%. Mesmo nas séries em que o teste ADF teria seu resultado comprometido, devido à presença de componentes sazonais ou de quebra estrutural, esses resultados foram confirmados por meio do teste HEGY, em que o teste de hipótese de raiz unitária não sazonal e com ciclos mensais, semestrais ou anuais, representados pelos coeficientes $\pi^{\prime} s$ e pelos testes conjuntos F's, não rejeitaram alguns testes de presença de raiz unitária e o teste de quebra estrutural, cuja hipótese de teste é presença de raiz unitária com quebra estrutural também não

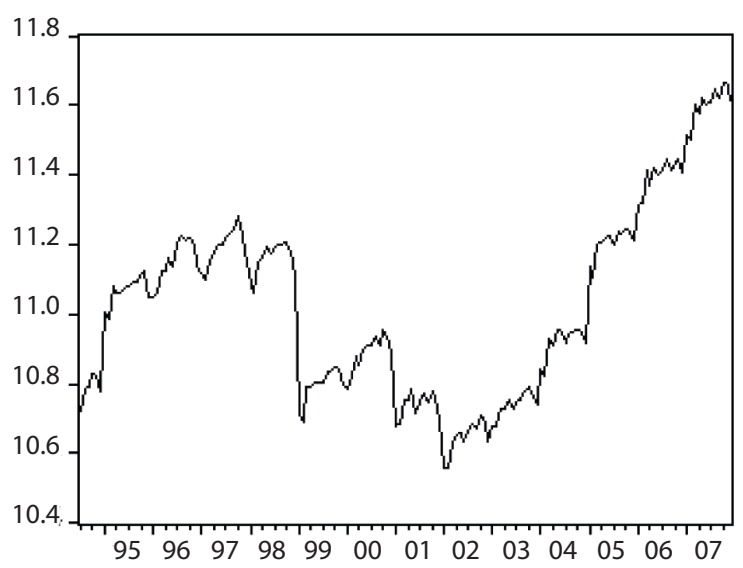

(b) $Y$

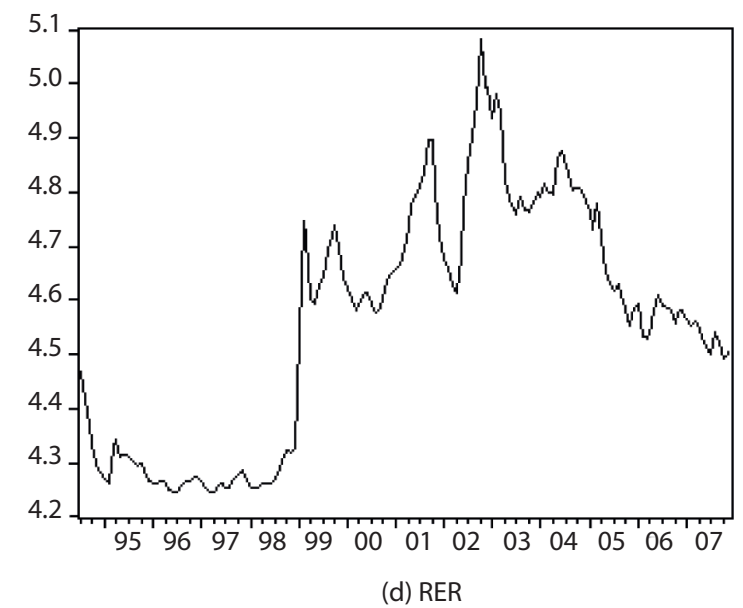

é significativo, ou seja, a hipótese nula não é rejeitada. Dessa forma, pode-se inferir que todas as séries são não estacionárias em nível.

O passo seguinte foi realizar o mesmo procedimento para as variáveis em primeira diferença (representadas pela letra do alfabeto grego $\Delta$ ). De acordo com o teste ADF, a hipótese nula de raiz unitária é rejeitada para as variáveis $\Delta \ln B P, \Delta \ln Y$ e $\Delta \ln R E R$ e não rejeitada apenas para $\Delta \ln Y^{*}$. Entretanto, por meio do teste de HEGY, rejeita-se todos os testes de raiz unitária não sazonal e com ciclos sazonais; para a variável $\Delta \ln Y^{*}$, este fato confirma a premissa de que a presença de componentes sazonais prejudica o 
Tabela 1. Testes de raiz unitária para variáveis $\ln (\mathrm{BP}), \ln (\mathrm{Y}), \ln \left(\mathrm{Y}^{*}\right)$ e $\ln (\mathrm{RER})$ em nível e primeira diferença $(\Delta)$

\begin{tabular}{|c|c|c|c|c|c|c|c|c|c|c|c|c|c|}
\hline & \multicolumn{3}{|c|}{ Teste ADF } & \multicolumn{9}{|c|}{ Teste HEGY } & \multirow{2}{*}{$\begin{array}{c}\text { Quebra } \\
\text { Estrutural }\end{array}$} \\
\hline & $\tau_{t}$ & $\tau_{m}$ & $\tau$ & $\pi_{1}^{*}$ & $\pi_{2}^{*}$ & $F 34^{¥}$ & $F 56^{¥}$ & $F 78^{¥}$ & $F 910^{¥}$ & $F 1112^{¥}$ & $F 1-12^{¥}$ & $F 2-12^{*}$ & \\
\hline $\ln \mathrm{BP}$ & -2.21 & -0.88 & 1.08 & 0.75 & $-3.30^{* * *}$ & $9.05^{* * *}$ & $7.86^{* * *}$ & 1.37 & $8.70^{* * *}$ & 2.19 & $6.12^{* * *}$ & $6.60^{* * *}$ & \\
\hline $\ln \mathrm{Y}$ & -0.99 & -0.77 & 1.16 & & & & & & & & & & -0.34 \\
\hline $\ln Y^{*}$ & -0.98 & 1.83 & 2.33 & 1.19 & -0.97 & 0.29 & $4.18^{* *}$ & $8.84^{* * *}$ & $5.68^{* * *}$ & 1.84 & $5.15^{* * *}$ & $5.26^{* * *}$ & \\
\hline ln RER & -1.36 & -1.48 & 0.14 & & & & & & & & & & 0.78 \\
\hline$\Delta \ln \mathrm{BP}$ & $-10.31^{* * *}$ & & & $-4.28^{* * *}$ & $-2.83^{* * *}$ & $5.05^{* * *}$ & $9.49^{* * *}$ & 1.239 & $9.02^{* * *}$ & $3.16^{* *}$ & $8.50^{* * *}$ & $6.57^{* * *}$ & \\
\hline$\Delta \ln Y$ & $-12.95^{* * *}$ & & & & & & & & & & & & $-7.91^{* * *}$ \\
\hline$\Delta \ln Y^{*}$ & -2.47 & -1.87 & -1.17 & $2.23^{* *}$ & $2.74^{* * *}$ & $3.67^{* * *}$ & $9.72^{* * *}$ & $7.30^{* * *}$ & $8.49^{* * *}$ & $7.32^{* * *}$ & $3.93^{* * *}$ & $4.17^{* * *}$ & \\
\hline$\Delta \ln$ RER & $-8.86^{* * *}$ & & & & & & & & & & & & $-6.95^{* * *}$ \\
\hline
\end{tabular}

valor do teste $\mathrm{ADF}$, comprometendo seu resultado. Além disso, a hipótese de presença de raiz unitária é rejeitada para os testes de quebra estrutural nas variáveis $\Delta \ln Y$ e $\Delta \ln R E R$. Portanto, pode-se inferir que todas as variáveis são estacionárias em primeira diferença, determinando seu grau de integração, ou seja, pode-se concluir que todas as variáveis utilizadas no modelo são I(1).

\subsection{Testes de cointegração de Johansen}

Os resultados dos testes de raiz unitária sugerem que um modelo VEC seria mais apropriado que um modelo VAR para caracterizar as relações multivariadas entre as quatro séries utilizadas (HAMILTON, 1994). Um VEC é um VAR na forma de primeira-diferença, embutindo explicitamente uma cointegração que captura a informação de longo prazo contida na tendência estocástica de cada série utilizada. Segundo o método, do ponto de vista econômico, quando duas ou mais séries estão cointegradas, as mesmas se movem conjuntamente no tempo e suas diferenças são estáveis (estacionárias), mesmo quando cada série em particular tem uma tendência estocástica e seja, portanto, não estacionária. A cointegração reflete a presença de um equilíbrio de longo prazo para o qual o sistema econômico converge.

Seguindo Hamilton (1994), se cada série é um processo I(1), a possibilidade de equilíbrio é analisada utilizando-se o teste de cointegração de Johansen (1991). Estes resultados estão reportados na Tabela 2.

$\mathrm{Na}$ estimativa dos vetores de cointegração é importante selecionar uma defasagem ótima que irá gerar resíduos do tipo ruído branco (White Noise). Esta etapa é muito importante na análise, uma vez que as defasagens exercem influência significativa sobre os resultados. Desta forma, foram utilizados os testes estatísticos de razão de probabilidade sequencial modificada (LR), erro de previsão final (FPE) e o critério de Akaike (AIC) para determinar uma defasagem de sete períodos, ou seja, $\mathrm{K}=7$.

O VEC associado aos resultados do teste na Tabela 2 foi estimado com seis lags de defasagem. Cada equação de cointegração contém intercepto sem tendência determinística. $\mathrm{O}$ resultado dos testes estatísticos do traço $\left(\lambda_{\text {Traço }}\right)$ e o teste da raiz 
Tabela 2. Teste de Johansen para vetor de cointegração

\begin{tabular}{|c|c|c|c|c|c|}
\hline \multicolumn{6}{|c|}{ Teste do Traço } \\
\hline \multicolumn{2}{|c|}{ Teste de hipótese } & \multirow{2}{*}{ Raiz característica } & \multirow{2}{*}{ Traço Calculado } & \multirow{2}{*}{ Valor crítico (5\%) } & \multirow{2}{*}{ Prob. * } \\
\hline $\mathrm{H} 0$ & $\mathrm{H} 1$ & & & & \\
\hline$r=0$ & $r \geq 1$ & 0,30 & 98,33 & 47,86 & 0,000 \\
\hline $\mathrm{r} \leq 1$ & $r \geq 2$ & 0,19 & 42,51 & 29,80 & 0,001 \\
\hline$r \leq 2$ & $r \geq 3$ & 0,04 & 7,98 & 15,49 & 0,468 \\
\hline$r \leq 3$ & $r \geq 4$ & 0,00 & 0,39 & 3,84 & 0,535 \\
\hline \multicolumn{6}{|c|}{ Teste da Raiz Característica Máxima } \\
\hline \multicolumn{2}{|c|}{ Teste de hipótese } & couractoríctica & rastarística máxima & tipo $(501)$ & 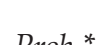 \\
\hline $\mathrm{HO}$ & $\mathrm{H} 1$ & Nata cuructertstica & 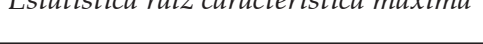 & (IILC $(5 \%)$ & 1700. \\
\hline $\mathrm{r}=0$ & $r=1$ & 0,30 & 55,82 & 27,58 & 0,000 \\
\hline$r \leq 1$ & $r=2$ & 0,19 & 34,53 & 21,13 & 0,000 \\
\hline$r \leq 2$ & $r=3$ & 0,04 & 7,59 & 14,26 & 0,421 \\
\hline$r \leq 3$ & $\mathrm{r}=4$ & 0,00 & 0,39 & 3,84 & 0,534 \\
\hline
\end{tabular}

r é o rank de cointegração; * Valores críticos de MacKinnon-Haug-Michelis (1999).

característica máxima $\left(\lambda_{\text {Máx }}\right)$, ao nível de $5 \%$ de significância, rejeitam a hipótese de $r=0, r \leq 1$ para o teste do traço e de raiz característica máxima. Entretanto, falham ao rejeitar as hipóteses de que o rank de cointegração do sistema é $\mathrm{r} \leq 2$. Assim, pode-se concluir que existem duas combinações lineares, estacionárias: uma combinação linear entre $(B P),\left(Y^{*}\right)$ e $(R E R)$ e outra, entre $(Y),\left(Y^{*}\right)$ e (RER).

\subsection{Modelo Vetor de Correção do Erro}

Como encontrado até aqui, a abordagem mais adequada para se quantificar as relações entre série I(1) é construir um modelo VEC. Neste modelo, a primeira diferença de cada variável é representada como uma função de seus próprios valores defasados, na ordem de seis defasagens $(\mathrm{K}-1=6)$ e duas equações de cointegração. $\mathrm{O}$ modelo VEC utilizado para conduzir os testes de cointegração na Tabela 3 é dado pelas seguintes equações ${ }^{5}$ :

5 A ordem de inclusão das variáveis no modelo VEC segue a especificação da forma empírica da eq. (11), que, por sua vez, foi determinada de acordo com a tradição encontrada na literatura empírica. Alguns exemplos são: Bahmani-Oskooee et al. (1998), Gupta-Kappor et al. (1999), Arora et al. (2003), Kamoto (2006), Bahmani-Oskooee et al. (2006) e Ardalani (2007).

$$
\begin{aligned}
& \Delta B P=\sum_{j=1}^{k}\left(\begin{array}{l}
\alpha_{1 j} \Delta B P_{t-j}+\beta_{1 j} \Delta Y_{t-j}+ \\
+\phi_{1 j} \Delta Y^{*}{ }_{t-j}+\gamma_{1 j} \Delta R E R_{t-j}
\end{array}\right) \\
& +\lambda_{11}\left(\tilde{\varepsilon}_{1 t-1}\right)+\lambda_{12}\left(\tilde{\varepsilon}_{2 t-1}\right)+\varepsilon_{1 t} \\
& \Delta Y=\sum_{j=1}^{k}\left(\begin{array}{l}
\alpha_{2 j} \Delta B P_{t-j}+\beta_{2 j} \Delta Y_{t-1}+ \\
+\phi_{2 j} \Delta Y^{*}{ }_{t-j}+\gamma_{2 j} \Delta R E R_{t-j}
\end{array}\right) \\
& +\lambda_{21}\left(\tilde{\varepsilon}_{1 t-1}\right)+\lambda_{22}\left(\tilde{\varepsilon}_{2 t-1}\right)+\varepsilon_{1 t} \\
& \Delta Y^{*}=\sum_{j=1}^{k}\left(\begin{array}{l}
\alpha_{3 j} \Delta B P_{t-j}+\beta_{3 j} \Delta Y_{t-j}+ \\
+\phi_{3 j} \Delta Y^{*}{ }_{t-j}+\gamma_{3 j} \Delta R E R_{t-j}
\end{array}\right) \\
& +\lambda_{31}\left(\tilde{\varepsilon}_{1 t-1}\right)+\lambda_{32}\left(\tilde{\varepsilon}_{2 t-1}\right)+\varepsilon_{1 t} \\
& \triangle R E R=\sum_{j=1}^{k}\left(\begin{array}{l}
\alpha_{4 j} \Delta B P_{t-j}+\beta_{4 j} \Delta Y_{t-j}+ \\
+\phi_{4 j} \Delta Y^{*}{ }_{t-j}+\gamma_{4 j} \Delta R E R_{t-j}
\end{array}\right) \\
& +\lambda_{41}\left(\tilde{\varepsilon}_{1 t-1}\right)+\lambda_{42}\left(\tilde{\varepsilon}_{2 t-1}\right)+\varepsilon_{1 t}
\end{aligned}
$$

em que $t$ representa meses, $\triangle B P$ é a primeira diferença do logaritmo natural da balança comercial agropecuária, $\Delta Y$ é a primeira diferença do logaritmo natural do PIB, $\Delta Y^{*}$ é a primeira diferença do logaritmo natural das importações mundiais (renda externa) e $\triangle R E R$ é a primeira diferença do logaritmo natural da taxa de câmbio real; $\tilde{\varepsilon}_{1 t-1}=B P_{t-1}-\theta_{1} Y^{*}{ }_{t-1}-\delta_{1} R E R_{t-1}-\pi_{1} \mathrm{e}$ $\varepsilon_{2 t-1}=Y_{t-1}-\theta_{2} Y^{*}{ }_{t-1}-\delta_{2} R E R_{t-1}-\pi_{2}$ são os resíduos de desequilíbrio, defasados um mês, provenientes das respectivas equações de cointegração; 
Tabela 3. Parâmetros estimados para os vetores de cointegração normalizados

\begin{tabular}{cccccc}
\hline Eq. de cointegração & $B P_{c t-1}$ & $Y_{m t-1}$ & $Y_{\mathrm{t}-1}^{*}$ & $R E R_{\mathrm{t}-1}$ & $\mathrm{C}$ \\
\hline \multirow{2}{*}{$\tilde{\varepsilon}_{1 t}$} & 1 & 0 & $-1.95^{* * *}$ & $-2.04^{* * *}$ & 34.12 \\
& & & $(0.150)$ & $(0.208)$ & \\
$\tilde{\varepsilon}_{2 t}$ & 0 & \multirow{2}{*}{1} & $-0.74^{* * *}$ & $1.16^{* * *}$ & -6.41 \\
\hline
\end{tabular}

* significativo a $10 \%,{ }^{* *}$ significativo a $5 \% \mathrm{e}^{* * *}$ significativo a $1 \%$.

$\alpha, \beta, \phi, \gamma, \lambda, \theta, \delta$ e $\pi$ são parâmetros desconhecidos a serem estimados e $\varepsilon$ representa um vetor de erros estocásticos usual.

Em tal sistema, quanto aos parâmetros $\alpha, \beta$, $\theta$ e $\gamma$, é difícil de serem determinados os sinais esperados a priori e o mesmo se aplica à constante $\pi$. Entretanto, espera-se que $\theta>0$ e $\delta>0$, o que implica que aumentos na renda do exterior e desvalorizações cambiais tenham impactos positivos tanto na balança comercial agropecuária $(B P)$ quanto na renda doméstica ( $Y$ ). Além disso, os parâmetros $\lambda$ representam as velocidades de ajustamento de desequilíbrios que ocorrem no curto prazo até restabelecer o nível de longo prazo.

Na estimação do VEC, como já apresentado, foi utilizado o teste estatístico de razão de probabilidade sequencial modificada (LR), erro de previsão final (FPE) e o critério de Akaike (AIC) para determinar a defasagem de sete períodos, ou seja, $\mathrm{K}=7$. De acordo com os testes do Multiplicador de Lagrange, para correlação serial nos resíduos, ao nível de 1\% de significância, foram rejeitadas as hipóteses de correlação serial. O teste de normalidade dos resíduos, por meio do método de Cholesky de ortogonalização dos resíduos, rejeitou a hipótese nula de que os resíduos sejam normalmente distribuídos. Os valores de $R^{2}$ variam entre 0,30 e 0,60, indicando que o modelo explica, relativamente bem, um grande percentual da variação da mudança do logaritmo natural de cada série econômica.

A Tabela 3 reporta as estimativas empíricas de $\theta, \delta$ e $\pi$ para os dois vetores de cointegração normalizados. Esses resultados, como discutido, representam as relações de longo prazo entre $(B P),\left(Y^{*}\right)$ e $(R E R)$ e entre $(Y),\left(Y^{*}\right)$ e $(R E R)$. Os parâmetros $\theta$ e $\delta$ correspondem com o esperado a priori para o primeiro vetor de cointegração
(BP), ou seja, são positivos (como os vetores estão normalizados, em sua interpretação torna-se necessário inverter os sinais) e foram estatisticamente significativos a $1 \%$. Uma vez estabelecida a existência de um equilíbrio de longo prazo entre $\mathrm{BP}$ e as duas variáveis, isto significa que um aumento de $1 \%$ na renda estrangeira e da taxa de câmbio real, implica aumentos de 1,95\% e 2,04\%, respectivamente, na razão da balança comercial agropecuária brasileira.

Para o segundo vetor de cointegração $(Y)$, apenas o parâmetro $\theta$, com sinal positivo, correspondeu com o esperado a priori. $\delta$ apresentou sinal negativo, além disso, ambos foram estatisticamente significativos a $1 \%$. Novamente, dada a relação de equilíbrio de longo prazo, pode-se inferir que um aumento de $1 \%$ na renda estrangeira implica, no longo prazo, uma elevação de $0,74 \%$ na renda doméstica e uma alta de $1 \%$ na taxa de câmbio real, ocasiona, no longo prazo, uma redução de 1,16\% na renda doméstica.

As estimativas empíricas das velocidades de ajustamento, ou os parâmetros $\lambda \mathrm{s}$, estão resumidos na Tabela 4. A priori, interessa analisar os parâmetros $\lambda_{11}$ e $\lambda_{22}$, que representam como os desequilíbrios transitórios (de curto prazo), que desviam as variáveis $(B P)$ e $(Y)$ de suas trajetórias de longo prazo, são corrigidos por meio dos vetores de cointegração. Os valores negativos obtidos $\left(\lambda_{11}=-0,731\right.$ e $\left.\lambda_{22}=-0,139\right)$ significam que choques positivos requerem que tanto $(B P)$ quanto $(Y$ ) caiam, com o passar do tempo, para restabelecerem suas trajetórias de equilíbrio de longo prazo. O coeficiente $\lambda_{11}$ mostrou-se estatisticamente significativo ao nível de $1 \%$ de significância, enquanto o coeficiente $\lambda_{12}$ é significativo apenas ao nível de $10 \%$. Isso implica que, dado um choque no curto prazo, que tira 
Tabela 4. Parâmetros estimados do modelo de vetor de correção de erros (VEC).

\begin{tabular}{|c|c|c|c|c|}
\hline Variáveis & $\Delta \mathrm{BP}$ & $\Delta \mathrm{Y}$ & $\Delta \mathrm{Y}^{*}$ & $\Delta \mathrm{RER}$ \\
\hline$\tilde{\varepsilon}_{1 t-1}$ & $-0.731^{* * *}$ & -0.022 & 0.014 & -0.001 \\
\hline$\tilde{\varepsilon}_{2 t-1}$ & $-1.517^{* * *}$ & $-0.139^{*}$ & $0.119^{*}$ & $-0.325^{* * *}$ \\
\hline$\Delta \mathrm{BP}_{-1}$ & $0.338^{* * *}$ & $0.029^{* *}$ & -0.008 & 0.002 \\
\hline$\Delta \mathrm{BP}_{-2}$ & $0.506^{* * *}$ & $0.034^{* * *}$ & $0.028^{* * *}$ & 0.000 \\
\hline$\Delta \mathrm{BP}_{-3}$ & $0.441^{* * *}$ & 0.011 & -0.008 & -0.005 \\
\hline$\Delta \mathrm{BP}_{-4}$ & $0.277^{* * *}$ & $0.028^{* *}$ & -0.011 & -0.004 \\
\hline$\Delta \mathrm{BP}_{-5}$ & 0.104 & -0.008 & $-0.030^{* * *}$ & $0.017^{* *}$ \\
\hline$\Delta \mathrm{BP}_{-6}$ & -0.029 & $0.021^{*}$ & $0.017^{* *}$ & 0.004 \\
\hline$\Delta \mathrm{Y}_{-1}$ & 0.742 & 0.047 & -0.046 & 0.056 \\
\hline$\Delta \mathrm{Y}_{-2}$ & $1.121^{* *}$ & $0.138^{*}$ & $0.185^{* *}$ & $0.310^{* * *}$ \\
\hline$\Delta \mathrm{Y}_{-3}$ & $1.053^{*}$ & -0.003 & -0.053 & $0.135^{* *}$ \\
\hline$\Delta \mathrm{Y}_{-4}$ & -0.497 & -0.000 & -0.053 & 0.015 \\
\hline$\Delta \mathrm{Y}_{-5}$ & 0.049 & 0.096 & 0.026 & $0.084^{*}$ \\
\hline$\Delta \mathrm{Y}_{-6}$ & $1.031^{* *}$ & -0.084 & -0.054 & 0.065 \\
\hline$\Delta \mathrm{Y}^{*}{ }_{-1}$ & $-2.932^{* * *}$ & -0.136 & $-0.401^{* * *}$ & $-0.212^{* * *}$ \\
\hline$\Delta \mathrm{Y}^{*}{ }_{-2}$ & $-4.726^{* * *}$ & $-0.262^{* *}$ & $-0.452^{* * *}$ & $-0.172^{* *}$ \\
\hline$\Delta Y_{-3}^{*}$ & $-4.497^{* * *}$ & $-0.213^{*}$ & -0.116 & -0.104 \\
\hline$\Delta \mathrm{Y}^{*}{ }_{-4}$ & $-2.506^{* * *}$ & -0.067 & -0.142 & -0.083 \\
\hline$\Delta \mathrm{Y}^{*-5}$ & -0.852 & $0.193^{*}$ & 0.096 & -0.086 \\
\hline$\Delta Y^{*}{ }_{-6}$ & 0.684 & $0.216^{* *}$ & $0.320^{* * *}$ & $-0.139^{* *}$ \\
\hline$\Delta \mathrm{RER}_{-1}$ & 0.877 & 0.067 & $0.161^{*}$ & $0.594^{* * *}$ \\
\hline$\Delta \mathrm{RER}_{2}$ & $1.328^{*}$ & 0.015 & -0.026 & 0.099 \\
\hline$\Delta \mathrm{RER}_{3}$ & 0.971 & 0.003 & $-0.266^{* *}$ & 0.111 \\
\hline$\Delta \mathrm{RER}_{4}$ & $-1.865^{* *}$ & -0.114 & -0.083 & $0.200^{* *}$ \\
\hline$\Delta \mathrm{RER}_{5}$ & 0.141 & $0.192 *$ & 0.063 & $0.180^{* *}$ \\
\hline$\Delta \mathrm{RER}_{6}$ & 0.067 & -0.141 & $-0.220^{* *}$ & 0.032 \\
\hline $\mathrm{C}$ & $0.073^{* * *}$ & 0.002 & $0.013^{* * *}$ & 0.002 \\
\hline $\mathrm{R}^{2}$ & 0.606318 & 0.301944 & 0.603179 & 0.465192 \\
\hline $\mathrm{R}^{2}$ Ajustado & 0.526351 & 0.160152 & 0.522575 & 0.356559 \\
\hline F-statistic & 7.582129 & 2.129478 & 7.483216 & 4.282243 \\
\hline Log likelihood & -34.1528 & 252.0903 & 285.4692 & 322.9769 \\
\hline Determinant resid covariance & & $2.03 \mathrm{E}-10$ & & \\
\hline Log likelihood & & 850.0102 & & \\
\hline
\end{tabular}

* significativo a $10 \%,{ }^{* *}$ significativo a $5 \% \mathrm{e} * * *$ significativo a $1 \%$. 
as variáveis $(B P)$ ou $(Y)$ de suas trajetórias de longo prazo, aproximadamente $73 \%$ e $13,9 \%$ do desequilíbrio, respectivamente, são corrigidos de um período para outro.

\subsection{Relações dinâmicas de curto prazo}

A dinâmica do relacionamento das variáveis com a balança comercial agropecuária brasileira pode ser observada pela análise da decomposição da variância, que gera resultados que permitem analisar o percentual da variação do erro devido a choques ocorridos nela mesma, e em proporção das outras variáveis do modelo (NARAYAN e NARAYAN, 2004).

Podem ser observados na Tabela 5 os resultados da decomposição da variância do saldo da balança comercial atribuída à sua própria variação e aos choques nas outras variáveis em um horizonte estimado de 1 a 12 meses.

Os resultados da decomposição da variância sugerem que, para a balança comercial agropecuária, a fonte mais significativa da variação ao longo dos períodos são choques ocorridos na própria variável, que não são captados pelas outras variáveis do modelo. Observa-se que a proporção da variância devida à própria variável decresce ao longo do período, porém, no período estimado, a média permanece em torno de $71,52 \%$. A renda nacional explica, em média, 1,73\% da variação do saldo da balança comercial. Entretanto, as importações mundiais e a taxa de câmbio real representam, em média, 10,27\% e 16,48\%, respectivamente. Em geral, pode-se observar que, dada a pouca dependência de importações de produtos agropecuários, os principais fatores que determinam a variação de $\mathrm{BP}$ são fatores diretamente ligados à determinação das exportações do setor, ou seja, renda externa e a taxa de câmbio real.

As funções de impulso-resposta representam uma forma conveniente de apresentar os coeficientes estimados pelo VAR. Este é capaz de capturar as dinâmicas de curto prazo do saldo da balança comercial agropecuária brasileira em resposta a choques na taxa de câmbio real, permitindo, assim, verificar se existe o efeito da Curva J.

A Figura 3 a seguir mostra a resposta do saldo da balança comercial a distúrbios de magnitude iguais a um desvio padrão na taxa de câmbio real. Pode-se observar que a balança comercial aumenta após o choque até o terceiro mês, quando alcança um máximo de 0,04 desvio padrão, em função de uma depreciação da taxa de câmbio real.

Tabela 5. Decomposição da variância dos erros de previsão da balança comercial agropecuária, em porcentagem

\begin{tabular}{ccccc}
\hline Período & BP & Y & Y* $^{*}$ & RER \\
\hline 1 & 100.00 & 0.00 & 0.00 & 0.00 \\
2 & 97.79 & 0.22 & 0.11 & 1.88 \\
3 & 88.87 & 1.18 & 3.43 & 6.52 \\
4 & 80.25 & 1.68 & 5.10 & 12.97 \\
5 & 70.75 & 2.32 & 4.53 & 22.40 \\
6 & 66.08 & 2.39 & 6.13 & 25.41 \\
7 & 61.88 & 2.09 & 12.87 & 23.15 \\
8 & 59.32 & 1.99 & 16.66 & 22.02 \\
9 & 58.74 & 2.12 & 17.90 & 21.25 \\
10 & 58.53 & 2.10 & 18.50 & 20.86 \\
11 & 58.03 & 2.19 & 18.96 & 20.83 \\
Média & 57.95 & 2.48 & 19.07 & $\mathbf{1 0 . 2 7}$ \\
\hline
\end{tabular}


Figura 3. Resposta do saldo da balança comercial agropecuária a um distúrbio de um desvio padrão na taxa de câmbio real - 12 meses.

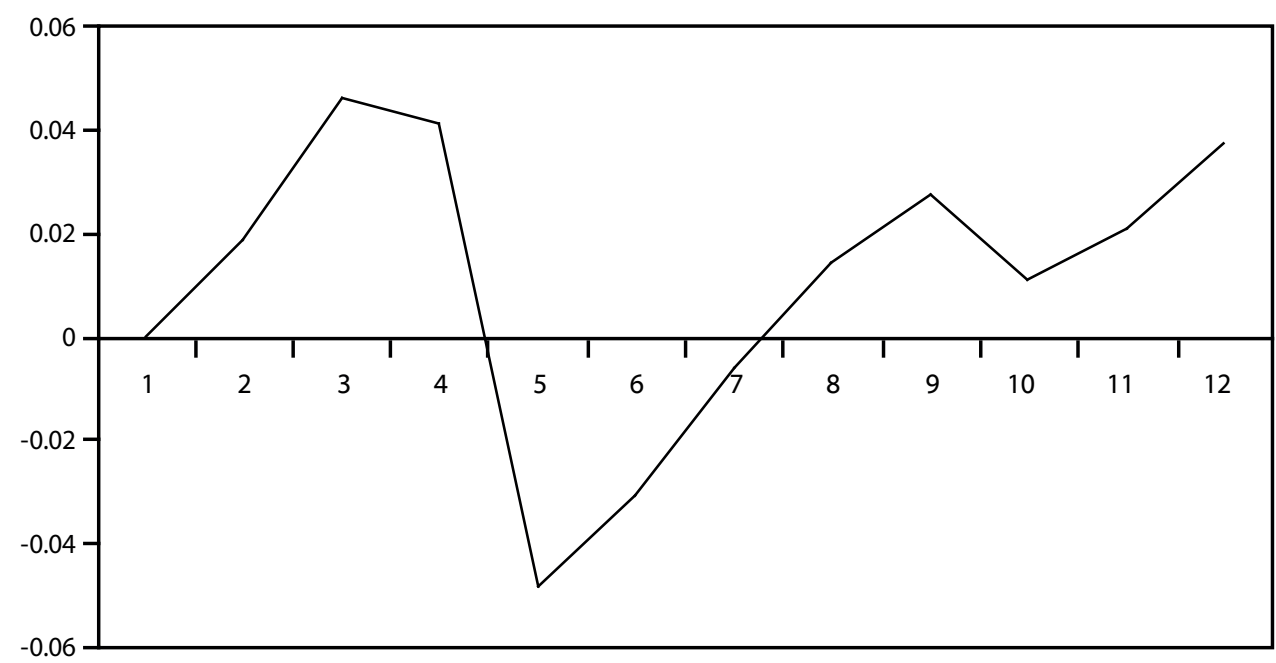

Após o terceiro mês, o saldo da balança comercial apresenta uma redução que prevalece somente até o quinto mês, a partir do qual é restabelecida uma trajetória ascendente, de forma cíclica, permanecendo num horizonte de tempo maior (aproximadamente 60 meses), em um nível maior do que antes, aproximadamente 0,02 desvio padrão (Figura 4).

Portanto, pode-se concluir, por meio da análise da função impulso-resposta, que a hipótese de existência do efeito Curva J, neste modelo de correção de erro linear, é rejeitada. No curto prazo, embora ocorra uma piora em BP, esta é antecedida por uma melhora de seu saldo e, após 12 meses, o choque acumulado é de 0,13 desvio padrão. No longo prazo, verifica-se o efeito da condição de Marsahll-Lerner, em que uma desvalorização cambial provoca um aumento do volume das exportações.

Figura 4. Resposta do saldo da balança comercial agropecuária a um distúrbio de um desvio padrão na taxa de câmbio real - 60 meses.

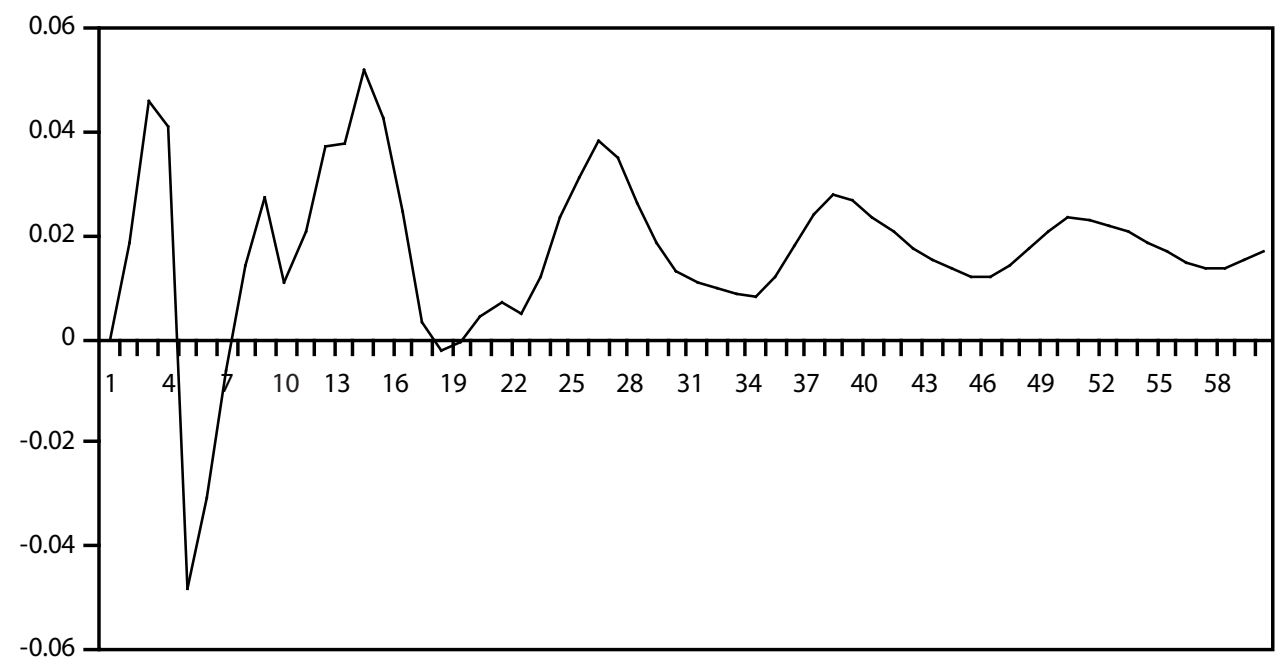




\section{Conclusão}

Os resultados indicam que existem duas relações de equilíbrio de longo prazo: a primeira, entre a balança comercial agrícola $(B P)$, renda externa $\left(Y^{*}\right)$ e taxa de câmbio real $(R E R)$, e a segunda, entre a renda doméstica $(Y)$, renda externa $\left(Y^{*}\right)$ e taxa de câmbio real (RER). Verifica-se que, no longo prazo, uma depreciação de $1 \%$ da taxa de câmbio ou um aumento de $1 \%$ na renda externa leva a aumentos de $2,04 \%$ e 1,95\%, respectivamente, no saldo da balança comercial do agronegócio. Esse resultado é consistente com a condição de Marshall-Lerner, ou seja, no longo prazo o efeito volume supera o efeito preço, o que aumenta o saldo da balança comercial.

Entretanto, no curto prazo, rejeita-se a hipótese de existência do efeito Curva J em que o saldo da balança comercial pioraria num primeiro instante, para melhorar somente depois de decorrido um determinado tempo. Assim, pode-se concluir que políticas cambiais de desvalorização da moeda nacional terão impactos positivos sobre as exportações do setor agropecuário brasileiro, tanto no curto quanto no longo prazo. Estas políticas serão bem sucedidas sob o ponto de vista de que não afetam negativamente o saldo da balança comercial.

Por fim, ressalta-se que alguns resultados encontrados dão indícios para a necessidade de novos estudos. Na segunda relação de equilíbrio encontrada, os resultados demonstram que um aumento de $1 \%$ na renda externa e uma desvalorização de $1 \%$ na taxa de câmbio real provocam efeitos contrários sobre a renda doméstica (aumento de 0,74\% e redução de $1,16 \%$, respectivamente). Portanto, parece existir um trade-off entre o saldo da balança comercial agropecuária e a renda doméstica, que deve ser considerado no que diz respeito à possibilidade de políticas cambiais. Além disso, a diferença de velocidade observada nos ajustes de distúrbios de curto prazo, no saldo da balança comercial e na renda doméstica dá indícios de que tais políticas podem ter efeitos distintos, no curto e no longo prazo, sobre as variáveis de interesse.

\section{Referências bibliográficas}

AHMAD, J.; YANG, J. Estimation of the J Curve in China. In: East-West Center. Working Papers, n. 67. disponível em: <http://www.eastwestcenter.org/ fileadmin/stored/pdfs/ECONwp067.pdf>, 2004.

ARDALANI, Z.; BAHMANI-OSKOOEE, M. Is there a J Curve at the Industry Level? Economics Bulletin, v. 6, n. 26, p. 1-12, 2007.

ARORA, S.; BAHMANI-OSKOOEE, M.; SWARNJIT, A. Bilateral J-Curve Between India and her Trading Partners. Journal of Applied Economics, v. 35, p. 1037-1041. 2003.

BAHMANI-OSKKOOEE, M.; NIROOMAND, F. Longrun price elasticities and the Marshall-Lerner condition revisited. Economics Letters, v. 61, n. 1, p. 101-109, 1998.

BAHMANI-OSKKOOEE, M.; ECONOMIDOU, C.; GOSWAMI, G. Bilateral J-curve between the UK vis-àvis her major trading partners. Applied Economics. v. 38, p. $879-888,2006$.

CARTER, C.; PICK, D. H. The J Curve Effect and the U.S. Agricultural Trade Balance. American Journal of Agricultural Economics, v. 71, n. 3, p. 712-720, 1989.

DOOROODIAN, K.; JUNG, C.; BOYD, R. The J Curve effect and US agricultural and industrial trade. Applied Economics, v. 31, n. 6, p. 687-695, 1999.

DOLDADO, J.; JENKINSON, T.; SOSVILLA-RIVERO, S. Cointegration and Unit Roots. Journal of Economic Survey, v. 4, p. 249-273, 1990.

ENDERS, W. Applied Econometric Time Series. New York: John Wiley, p. 433, 1995.

GOLDSTEIN, M.; KHAN M. S. Income and Price Effects of in Foreign Trade. In: Handbook of International Economics, v. 2, p. 1041-1105, 1985.

GUPTA-KAPOOR, A.; RAMAKRISHNA, U. Is There a J Curve? A New Estimation for Japan. International Economic Journal, v. 13, n. 4, p. 71-19, 1999.

HAMILTON, J. D. Time Series Analysis. Princeton: Princeton University Press, 1994.

JOHANSEN, S. estimation and hypothesis testing of cointegration vectors in Gaussian vector autoregressive models. Econometrica, v. 59, p. 1551-1580, 1991.

KAMOTO, E. B. The Curva J effect on the trade balance in Malawi and South Africa. 2006. Tese (Master of Arts in economics). The University of Texas at Arlington, Arlington. Disponível em: < http://economics.uta.edu/ theses/Eric.Kamoto.pdf $>$. 
KANNEBLEY, J. S. Desempenho exportador brasileiro recente e taxa de câmbio real: uma análise setorial. Revista Brasileira de Economia, v. 56, n. 3, p. 429-456, 2002.

LÜTKEPOHL, H.; KRÄTZIG, M. (Ed.). Applied time series econometrics. Cambridge: Cambridge University Press, p. 323, 2004.

MADALLA, G. S.; KIM, I. M. Unit roots, co-integration and structural change. Cambridge: Cambridge University Press, p. 505, 1998.
MOURA, G.; Da SILVA, S. Is there a Brazilian J Curve? Economic Bulletin, v. 6, n. 1, p. 1-17, 2005.

NARAYAN, P. K.; NARAYAN, S. The J-Curve: Evidence from Fiji. International Review of Applied Economics, Taylor and Francis Journals, v. 18, n. 3, p. 369-380, 2004.

YAZICI, M. Is the J Curve effect observable in Turkish agricultural sector? Journal of Central European Agriculture, v. 7, n. 2, p. 319-322, 2006. 
